\title{
Dynamics of Synthesis of Large Carbon Structures in the Interstellar Medium
}

\author{
N. Patra ${ }^{1}$ and H. R. Sadeghpour ${ }^{2}$ \\ ${ }^{1}$ Department of Chemistry, University of Illinois at Chicago, Chicago, IL 60607, USA \\ email: npatra2@uic.edu \\ ${ }^{2}$ ITAMP, Harvard-Smithsonian Center for Astrophysics, Cambridge, MA 02138, USA \\ email: hsadeghpour@cfa.harvard.edu
}

\begin{abstract}
We investigate the nucleation of carbon and hydrogen atoms in the gas phase to form large carbon chains, clusters and cages by reactive molecular dynamics simulations. We study how temperature, particle density, presence of hydrogen, and carbon inflow affect the nucleation of molecular moieties with different characteristics.
\end{abstract}

Keywords. ISM: molecules, ISM: molecular dynamics simulation, hydrogenation

\section{Introduction}

Carbon-based chemistry of the interstellar medium (ISM) is now an established discipline (Herbst \& Van Dishoeck 2009). Hydrocarbons, alcohols, acids, aldehydes, amines, and sugars have already been optically detected in the ISM (Kwok 2009, Hollis et al. 2000). Detection of ring species, including aromatic rings, raise the possibility that biologically significant molecules and polycyclic aromatic hydrocarbons (PAHs) (Tielens 2008) may exist in the ISM. The IR emission features at 7.0, 8.5, 17.4 and $18.9 \mu \mathrm{m}$, present in the spectra of Tc1, NGC 7023 and NGC 2023 planetary and reflection nebulae, have been associated with the vibrational lines of regular carbon cages $\left(\mathrm{C}_{60}\right.$ and $\left.\mathrm{C}_{70}\right)$ (Cami et al. 2010, Sellgren et al. 2010).

To our knowledge, there are no available dynamical simulations of formation of molecules with aromatic rings at astrophysically-relevant conditions. In this work, we use reactive molecular dynamics (MD) simulations to model the nucleation of carbon clusters, starting from randomly distributed elementary carbon and hydrogen atoms in a periodic box. We study how chains, rings, and cages of different sizes and moieties form in regimes relevant to astrophysics (cold temperatures and low densities). The MD simulations are carried out in thermodynamical conditions several orders of magnitude more dilute than those considered under laboratory conditions (Irle et al. 2003).

\section{Computational Method and Results}

We model the self-assembly of clusters from C-C, C-H, and $\mathrm{H}-\mathrm{H}$ bonds using atomistic reactive $\mathrm{MD}$ simulations with the adaptive intermolecular reactive empirical bondorder (AIREBO) potential (Stuart et al. 2000), as implemented in the LAMMPS package (Plimton 1995). The AIREBO potential function is proposed by Stuart (Stuart et al. 2000), based on the Brenner bond-order potential (Brenner 1992). This potential can be used for chemical reactions and intermolecular interactions in condensed-phase hydrocarbon systems such as liquids, graphite, and polymers. The systems are modeled in the NVE ensemble at targeted temperatures and the time step is 0.5 fs. The Langevin damping method (Servantie \& Gaspard 2003) is used to thermalize the systems and the 


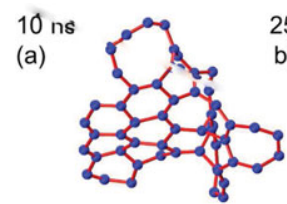

$25 \mathrm{~ns}$

b)

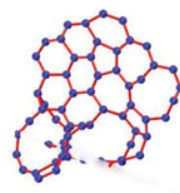

$50 \mathrm{~ns}$
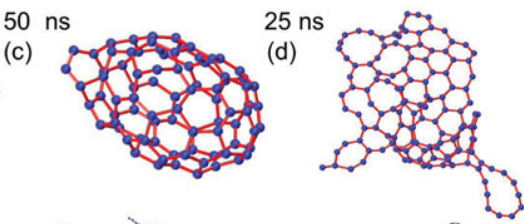

$50 \mathrm{~ns}$

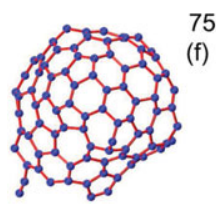

75 ns
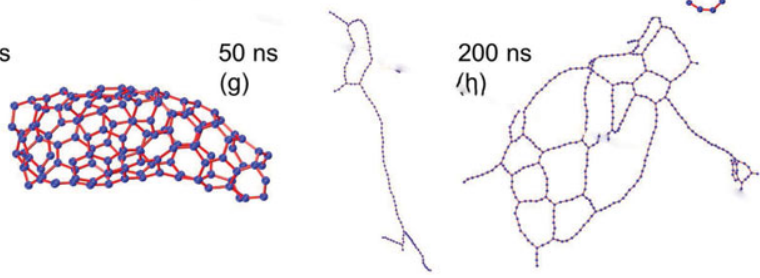

Figure 1. Self-assembly of carbon atoms at different temperatures. At $T=3000 \mathrm{~K}$ : (a) planar structures, (b) planar clusters with six and five member rings, (c) fullerene type clusters $\left(C_{70}-C_{80}\right)$. At $2000 \mathrm{~K}$ : (d) Planar structures, (e) cage/bowl like clusters, (f) Cylindrical clusters. At $500 \mathrm{~K}$ : (g) long chains, (h) planar clusters with large rings. (Snapshot times are to the left of each structure.)

damping co-efficient is $0.01 \mathrm{ps}^{-1}$. Periodic boundary conditions are applied and analysis of the trajectories are done by VMD (Humphrey et al. 1996).

In the actual simulations, 512 carbons atoms in the gas phase are placed in a cubic box with periodic boundary conditions. We change the density of carbon atoms $\left(4.1 \times 10^{-6}\right.$ $-5.8 \times 10^{-10} \AA^{-3}$ ) by varying the box size. We investigate the cluster formation over a range of temperatures. We also simulate two systems at different temperatures where the ratio between carbon atoms and hydrogen atoms are $1: 1$ and $1: 2$, respectively (in a $500 \times 500 \times 500 \AA^{3}$ box). Each MD simulation consists of two steps. First, the system energy is minimized for a short time $(5 \mathrm{ps})$, the systems are heated to the target temperature and equilibrated for up to $3 \mu \mathrm{s}$. Snapshots of carbon moieties formed at different times are taken for analysis and reproduction below (Patra et al. 2013).

\subsection{Formation of chain and cage structures}

MD simulations were performed at $T=3000,2000,1000,500$, and $300 \mathrm{~K}$. In a composite figure, Fig. 1, we give the time snapshots of carbon structure formation for a box size of $500 \times 500 \times 500 \AA^{3}$, giving a carbon particle density of $4.1 \times 10^{-6} \AA^{-3}$ at $T=3000,2000$ and $500 \mathrm{~K}$. At higher temperatures, as expected, diffusion and collision rates are faster and curvature forms at much earlier times.

In general, when the temperature is high enough to overcome the transition barriers, defects (five member rings) form and the cage like structures (bowls and fullerenes) begin to form. At lower temperatures, while there may be evidence for curvature formation, odd-atom number rings form but are irregular in shape.

Next, we study the effect of particle (carbon atom) density on the structure of carbon cages/clusters. Here, we simulated the clustering process with different carbon densities $\left(4.1 \times 10^{-6}-5.8 \times 10^{-10} \AA^{-3}\right)$ at $T=3,000 \mathrm{~K}$. When we decrease the concentration of carbon atoms $\left(\rho=5.1 \times 10^{-7} \AA^{-3}\right)$, short chain molecules emerge only after $100 \mathrm{~ns}$. After $450 \mathrm{~ns}$, long chain molecules with cage like clusters are observed. Fullerene type clusters are observed after $1 \mu \mathrm{s}$.

\subsection{Hydrogenation process}

We simulated two systems where the ratio between carbon and hydrogen atoms are $1: 1$ and $1: 2$, respectively. We also investigated the effect of concentration on the 
self-assembled structures by varying the atomic concentration. For each study, after a short minimization, we simulated the cluster formation at different temperatures $(T=$ $3000,2000,1000,500$, and $300 \mathrm{~K})$. Here, we notice the formation of alkene and other unsaturated carbon-hydrogen chain molecules along with small carbon clusters and hydrogen molecules.

\subsection{Carbon inflow: non-equilibrium nucleation}

We also plan to investigate the nucleation process of carbon atoms where carbon atoms are periodically added to the system, more realistically simulating the astrophysical environments. We will start to simulate with 100 gaseous carbon atom in a $150 \AA^{3}$ box at $1750 \mathrm{~K}$. After equilibrating for $10 \mathrm{~ns}, 20$ carbon atoms will be added into the box, keeping the box size fixed. After minimization, we will simulate for another $10 \mathrm{~ns}$ at 1750 $\mathrm{K}$ and save the coordinates of all atoms of the last frame and repeat the process again.

\section{Conclusion}

We demonstrate synthesis of large carbon structures in the gas phase in interstellar space by using reactive MD simulations. The influence of temperature, particle density and presence of hydrogen atoms on the shape of structures were studied. It is found that at high temperature $(2000-3000 \mathrm{~K})$ fullerene type clusters are obtained, whereas at relatively low temperature graphene type sheets along with long chain molecules/structures are synthesized. In presence of hydrogen atoms, fullerene type clusters are found at high temperature whereas short and branched chains molecules, terminated with hydrogen atoms, are observed at low temperature.

\section{Acknowledgements}

N.P. acknowledges the support from the SAO Fellowship. The authors are grateful for allocation of computer time on the Kraken Cluster at the NSF-XSEDE and Odyssey Clusters at Harvard University where parts of the simulations were conducted. Financial support was provided by a Smithsonian Grand Challenges Award.

\section{References}

Brenner, D. W. 1992, Phys. Rev. B, 42, 9458

Cami, J., Bernard-Salas, J., Peeters, E., \& Malek, S. E. 2010, Science, 329, 1180

Herbst, E. \& van Dishoeck, E. F. 2009, ARAESA, 47, 427

Hollis, J. M., Lovas, F. J., \& Jewell, P. R. 2000, ApJ, 540, L107

Humphrey, W., Dalke, A., \& Schulten, K. 1996, J. Mol. Graph., 14, 33

Irle, S., Zheng, G., Elstner, M., \& Morokuma, K. 2003, Nano Letters, 3, 1657;

Kwok, S. 2009, Astrophy. Space Sci., 319, 5

Patra, N., Král, P., \& Sadeghpour, H. R. 2014, in preparation

Sellgren, K. et al. 2010, ApJ Letters, 722, L54

Servantie, J. \& Gaspard, P. 2003, Phys. Rev. Lett., 91, 185503

Stuart, S. J., Tutein, A. B., \& Harrison, J. A. 2000, J. Chem. Phys., 112, 6472

Tielens, A. G. G. M. 2008, ARAEBA, 46, 289 